\title{
Correction: EGFR and COX-2 protein expression in non-small cell lung cancer and the correlation with clinical features
}

\author{
Feng Li ${ }^{1}$, Yongmei Liu', Huijiao Chen², Dianying Liao ${ }^{2}$, Yali Shen ${ }^{1}$, Feng $\mathrm{Xu}^{1{ }^{* \dagger}}$, Jin Wang ${ }^{\text {* }^{*}}$
}

\section{Correction}

In the article [1] there were errors in Tables three, four, five, six and seven. The incorrect values were produced due to typographical errors during translation stage. These errors affect neither the published discussion nor the conclusions of the paper. However, a few changes to the results section are detailed here.

\section{In the Abstract, under "Results" the first two sentences read}

"The positive rate of EGFR protein in NSCLC tumor cells was $46 \%$, which was significantly higher than its expression in normal lung $(\mathrm{p}=0.0234)$ and paracancerous tissues $(p=0.020)$. EGFR expression was significantly higher in nodal positive than in nodal negative patients $(\mathrm{p}=0.04)$."

But should have been:

"The positive rate of EGFR protein in NSCLC tumor cells was $46 \%$, which was significantly higher than its expression in normal lung $(\mathrm{p}=0.034)$ and paracancerous tissues $(p=0.020)$. EGFR expression was significantly higher in nodal positive than in nodal negative patients $(\mathrm{p}=0.006) . "$

\section{In the main "Results" section of the article}

The sentence under the heading "EGFR protein expression" read: "The positive rate of EGFR protein in NSCLC tumor cells were $46 \%$, which was significantly higher than its expression in normal lung $(\mathrm{p}=0.0234)$ and paracancerous $(\mathrm{p}=0.020)$ "
Which should have been:

"The positive rate of EGFR protein in NSCLC tumor cells were $46 \%$, which was significantly higher than its expression in normal lung ( $\mathrm{p}=0.034)$ and paracancerous $(\mathrm{p}=0.020)$ "

Under the heading "Correlation between EGFR expression and clinical features" The second sentence read: "It shows that the difference of EGFR expression was only significant between the nodal positive and negative subgroups $(56.4 \%$ vs. $10 \%, \mathrm{p}=0.04)$."

But the passage should have been "The expression of EGFR in different subgroups were compared and summarized in Table three. It shows that the difference of EGFR expression was only significant between the nodal positive and negative subgroups $(56.4 \%$ vs. $9.1 \%$, p $=$ 0.006). There is no significant difference between age (60 vs. under $60 \mathrm{ys}$ ), gender, adeno- vs. non-adenocarcinoma, the differentiation of tumor, and staging."

This is the correct table three (table 1).

Correct tables four (table 2), five (table 3) and six (table 4).

Under the heading "Correlation of EGFR and COX-2 expression" The sentence reads: "As shown in Table seven, no correlation was found between COX-2 and EGFR protein expression ( $\mathrm{X} 2=0.112, \mathrm{P}=0.555)$."

But should have read: "As shown in Table seven, no correlation was found between COX-2 and EGFR protein expression $(\mathrm{P}>0.05)$."

Correct table seven (Table 5).

* Correspondence: Fengxuster@gmail.com; jinwang593@yahoo.com.cn

† Contributed equally

'Radiation Oncology, Tumor Center, West China Hospital, Sichuan University,

PR China

Full list of author information is available at the end of the article

(C) $2011 \mathrm{Li}$ et al; licensee BioMed Central Ltd. This is an Open Access article distributed under the terms of the Creative Commons Attribution License (http://creativecommons.org/licenses/by/2.0), which permits unrestricted use, distribution, and reproduction in any medium, provided the original work is properly cited. 
Table 1 (corrected table 3). EGFR expression and clinical characteristics

\begin{tabular}{|c|c|c|c|c|}
\hline \multirow[t]{2}{*}{ Clinical features } & \multicolumn{2}{|c|}{ EGFR } & \multirow{2}{*}{$\begin{array}{c}\text { Positive } \\
\text { expression rate }\end{array}$} & \multirow{2}{*}{$\begin{array}{c}P \\
\text { value }\end{array}$} \\
\hline & negative & positive & & \\
\hline Ages & & & & 0.448 \\
\hline$<60$ & 18 & 14 & $43.80 \%$ & \\
\hline$\geq 60$ & 9 & 9 & $50 \%$ & \\
\hline Sex & & & & 0.445 \\
\hline Male & 16 & 15 & $48.40 \%$ & \\
\hline Female & 11 & 8 & $42.10 \%$ & \\
\hline Pathologic type & & & & 0.543 \\
\hline Squamous carcinoma & 13 & 8 & $38.10 \%$ & \\
\hline Adencarcinoma & 13 & 13 & $50.0 \%$ & \\
\hline Mixed type & 1 & 2 & $66.70 \%$ & \\
\hline Tumor length & & & & 0.535 \\
\hline$\leq 3 \mathrm{~cm}$ & 9 & 7 & $43.80 \%$ & \\
\hline$>3 \mathrm{~cm}$ & 18 & 16 & $47.10 \%$ & \\
\hline Level of Differentiation & & & & 0.474 \\
\hline Poor Differentiated & 6 & 4 & $40 \%$ & \\
\hline $\begin{array}{l}\text { Moderate and Well } \\
\text { Differentiated }\end{array}$ & 21 & 19 & $47.50 \%$ & \\
\hline TNM Stage & & & & 0.194 \\
\hline$|-| \mid$ & 10 & 5 & $33.30 \%$ & \\
\hline III-IV & 17 & 18 & $51.40 \%$ & \\
\hline Lymph node & & & & $0.006^{*}$ \\
\hline No & 10 & 1 & $9.10 \%$ & \\
\hline N1-3 & 17 & 22 & $56.40 \%$ & \\
\hline
\end{tabular}

*P $<0.05$.

Table 2 (corrected table four) COX-2 expression in neoplastic and normal tissue

\begin{tabular}{lccccc}
\hline Tissue type & $\begin{array}{c}\text { Number } \\
\text { of cases }\end{array}$ & \multicolumn{2}{c}{ COX-2 } & $\begin{array}{c}\text { Positive } \\
\text { rate(\%) }\end{array}$ & $\begin{array}{c}\mathrm{P} \\
\text { value }\end{array}$ \\
\cline { 3 - 4 } & & positive & negative & & \\
\hline $\begin{array}{c}\text { Neoplastic } \\
\text { tissue }\end{array}$ & 50 & 45 & 5 & 90 & $0.000^{*}$ \\
$\begin{array}{c}\text { Normal } \\
\text { tissue }\end{array}$ & 6 & 0 & 6 & 0 & \\
\hline $\mathrm{P}<0.05$. & & & & & \\
\end{tabular}

Table 3 (corrected table five) COX-2 expression in tumor and paracancerous tissue

\begin{tabular}{|c|c|c|c|c|c|}
\hline \multirow[t]{2}{*}{ Tissue type } & \multirow{2}{*}{$\begin{array}{l}\text { Number of } \\
\text { cases }\end{array}$} & \multicolumn{2}{|c|}{ COX-2 } & \multirow{2}{*}{$\begin{array}{l}\text { Positive } \\
\text { rate(\%) }\end{array}$} & \multirow{2}{*}{$\begin{array}{c}P \\
\text { value }\end{array}$} \\
\hline & & positive & negative & & \\
\hline $\begin{array}{l}\text { Neoplastic } \\
\text { tissue }\end{array}$ & 50 & 45 & 5 & 90 & $0.000^{*}$ \\
\hline $\begin{array}{l}\text { Paracancerous } \\
\text { tissue }\end{array}$ & 7 & 1 & 6 & 14.3 & \\
\hline
\end{tabular}

Table 4 (corrected table six) 6 COX-2 expression and correlation with clinical features

\begin{tabular}{|c|c|c|c|c|}
\hline \multirow[t]{2}{*}{ Clinical features } & \multicolumn{2}{|c|}{ COX-2 } & \multirow{2}{*}{$\begin{array}{c}\text { Positive } \\
\text { expression rate }\end{array}$} & \multirow{2}{*}{$\begin{array}{c}P \\
\text { value }\end{array}$} \\
\hline & negative & positive & & \\
\hline Ages & & & & 0.599 \\
\hline$\leq 60$ & 3 & 30 & $90.90 \%$ & \\
\hline$>60$ & 2 & 15 & $88.20 \%$ & \\
\hline Sex & & & & 0.362 \\
\hline Male & 4 & 27 & $87.10 \%$ & \\
\hline Female & 1 & 18 & $94.70 \%$ & \\
\hline Pathologic type & & & & $0.022^{*}$ \\
\hline Squamous carcinoma & 5 & 16 & $76.20 \%$ & \\
\hline Adencarcinoma & 0 & 26 & $100 \%$ & \\
\hline Mixed type & 0 & 3 & $100 \%$ & \\
\hline Tumor length & & & & 0.518 \\
\hline$\leq 3 \mathrm{~cm}$ & 2 & 14 & $87.50 \%$ & \\
\hline$>3 \mathrm{~cm}$ & 3 & 31 & $91.20 \%$ & \\
\hline Level of Differentiation & & & & 0.258 \\
\hline Poor Differentiated & 2 & 8 & $80 \%$ & \\
\hline $\begin{array}{l}\text { Moderate and Well } \\
\text { Differentiated }\end{array}$ & 3 & 37 & $92.50 \%$ & \\
\hline TNM Stage & & & & 0.476 \\
\hline$|-| \mid$ & 2 & 13 & $86.70 \%$ & \\
\hline III-IV & 3 & 32 & $91.40 \%$ & \\
\hline Lymph node & & & & 0.699 \\
\hline No & 1 & 10 & $90.90 \%$ & \\
\hline $\mathrm{N} 1-3$ & 4 & 35 & $89.70 \%$ & \\
\hline
\end{tabular}

$* P<0.05$

Table 5 (corrected table seven) Correlation of EGFR and COX-2 protein expression

\begin{tabular}{ccccc}
\hline & & \multicolumn{2}{c}{ EGFR } & \multirow{2}{*}{ Total } \\
\cline { 3 - 4 } & & negative & Positive & \\
\hline \multirow{2}{*}{ COX-2 } & negative & 3 & 2 & 5 \\
& positive & 24 & 21 & 45 \\
& Total & 27 & 23 & 50 \\
\hline
\end{tabular}

There was no significant relationship between COX-2 and EGFR. P > 0.05 .

Author details

${ }^{1}$ Radiation Oncology, Tumor Center, West China Hospital, Sichuan University, PR China. ${ }^{2}$ Department of Pathology, West China Hospital, Sichuan University, PR China.

Received: 28 March 2011 Accepted: 28 March 2011

Published: 28 March 2011

Reference

1. Li Feng, Liu Yongmei, Chen Huijiao, Liao Dianying, Shen Yali, Xu Feng, Wang Jin: EGFR and COX-2 protein expression in non-small cell lung cancer and the correlation with clinical features. Journal of Experimental \& Clinical Cancer Research 2011, 30:27.

doi:10.1186/1756-9966-30-32

Cite this article as: Li et al:: Correction: EGFR and COX-2 protein expression in non-small cell lung cancer and the correlation with clinical features. Journal of Experimental \& Clinical Cancer Research 2011 30:32. 